\title{
Validation and application of gas radiation computation at earth re-entry conditions
}

Cite as: AIP Conference Proceedings 2132, 180012 (2019); https://doi.org/10.1063/1.5119670

Published Online: 05 August 2019

Junming Lyu, Xiaoli Cheng, Jijun Yu, Fei Li, and Xilong Yu

ARTICLES YOU MAY BE INTERESTED IN

Assessment of recent thermo-chemical relaxation models using the DLR-TAU code AIP Conference Proceedings 2132, 140006 (2019); https://doi.org/10.1063/1.5119640

Impact of flow non-equilibrium in oxygen shock absorption analysis

AIP Conference Proceedings 2132, 180008 (2019); https://doi.org/10.1063/1.5119666

Preface: 31st International Symposium on Rarefied Gas Dynamics

AIP Conference Proceedings 2132, 010001 (2019); https://doi.org/10.1063/1.5119521

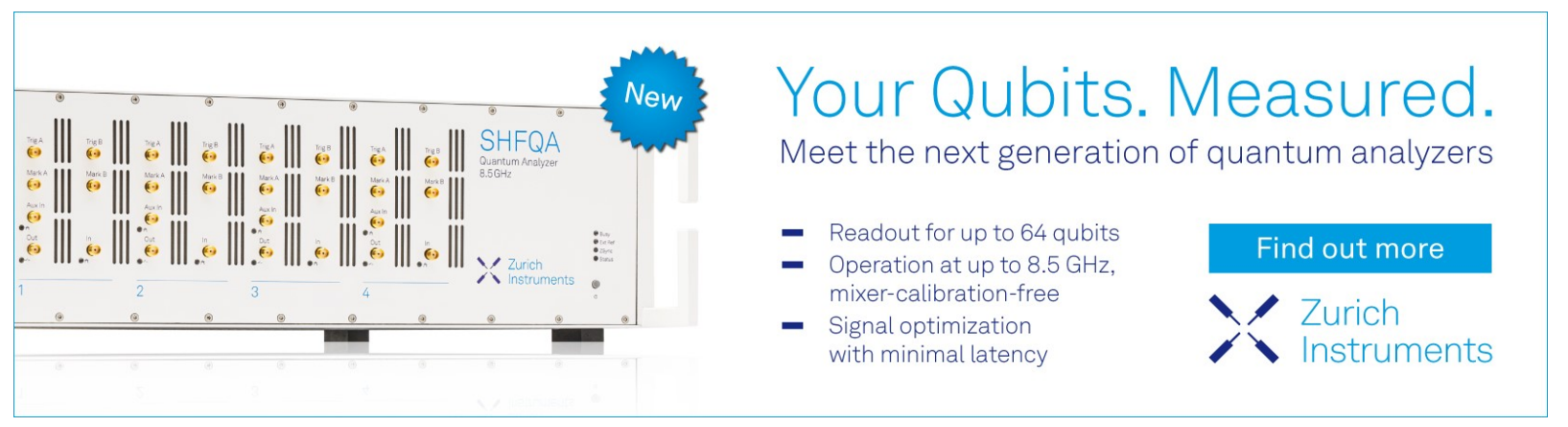




\title{
Validation and Application of Gas Radiation Computation at Earth Re-entry Conditions
}

\author{
Junming $\mathrm{Lyu}^{1, \text { a) }}$ Xiaoli Cheng ${ }^{1)} \mathrm{Jijun} \mathrm{Yu}^{1)} \mathrm{Fei}^{2}{ }^{2)}$ and Xilong $\mathrm{Yu}^{2)}$ \\ ${ }^{1}$ China Academy of Aerospace Aerodynamics, No.17 Yungangxi Road, Beijing, 100074, China \\ ${ }^{2}$ Key Laboratory of High Temperature Gas Dynamics, Institute of Mechanics, CAS, No. 15 Beisihuanxi Road, \\ Beijing, 100190, China \\ ${ }^{a)}$ Corresponding author: junminglyu@foxmail.com
}

\begin{abstract}
When vehicles re-enter the Earth's atmosphere at hyper-velocity, gas radiative heat will increase dramatically, even larger than convective heat. To reduce the design margin of thermal protection system, radiative heat must be considered and predicted accurately. Firstly, experimental tests of absolute gas radiative intensity measurement have been conducted in a combustion driven shock tube to validate the computational models and methods. The absolute value of gas spectral emission radiation in the spectrum range of 330 to $370 \mathrm{~nm}$ has been obtained for air at the velocity up to $8 \mathrm{~km} / \mathrm{s}$. Secondly, Unsteady Navier-Stokes equations with thermo-chemical non-equilibrium models, narrow-band radiation model accounting for both electronic and vibrational-rotational transition, and radiation transfer equation have been respectively solved to get the flow parameters and spectral radiant intensity at corresponding test condition with high spectrum resolution. The comparison between experimental and computational results showed an excellent agreement. Finally, numerical simulations on an Apollo-like re-entry vehicle FIRE II have been implemented. The results showed that radiative heating rate integrated in solid angle space and spectral interval were approximately the same for two catalytic wall conditions. Radiative heating rate decreased slowly and monotonously along the radial direction, while convective heat rose up again on the shoulder. The total heating rate of non-catalytic and super-catalytic wall covered the flight data.
\end{abstract}

\section{INTRODUCTION}

When vehicles re-enter Earth's atmosphere at hyper-velocity, high temperature gas in shock layer undergoes strong physical and chemical change, along with substantive radiation. Radiative heating will increase extremely fast along with the velocity goes up, may even be greater than convective heat at the velocity above $10 \mathrm{~km} / \mathrm{s}^{[1]}$. It is essential to predict gas radiation accurately to guarantee thermal protection system.

To model and simulate gas radiation correctly, experiment data is important. But flight tests for radiation measurement are difficult, expensive and limited. FIRE II project ${ }^{[2,3]}$ is the first success flight experiment for radiation measurement in which radiative and total heat flux were directly measured using built-in sensors. After FIRE II, there are several flight tests accomplished for thermal protection system under severe conditions of hyperbolic re-entry, such as Stardust in $2006^{[4]}$ and Hayabusa in $2010^{[5]}$. However, in these tests only indirect optical measurements have been executed. Till now, FIRE II test data is still the best candidate benchmark for radiation solver validation.

As to ground experiment, it is hard to conduct the test and measure radiative flux for small test models. Fortunately, in some high enthalpy facilities, several basic mechanism experiments counting for chemical reactions and radiation can be carried out to validate the models and methods ${ }^{[6]}$. In recent ten years, a number of tests for absolute radiation measurement in Earth re-entry environment have been implemented in EAST of NASA ${ }^{[7,8]}$, that has given a lot of 
data to correct the chemical reaction and radiation models. Besides, there are also other similar experiments taken in expansion tube X2 of Queensland University ${ }^{[9]}$ and shock tube HVST of JAXA Chofu Space Center ${ }^{[10]}$.

In this paper, experiments of measuring absolute spectral emission radiation of air at the velocity up to $8 \mathrm{~km} / \mathrm{s}$ has been conducted in a combustion driven shock tube. The volumetric spectral radiation in the spectrum range of 330 to $370 \mathrm{~nm}$ have been acquired. The simulations have been carried out using an in-house built code to solve the models and equations of gas dynamics and radiation. The comparison between the computation and experiment helped to validate the reaction and radiation models. Based on the validation, radiative and convective heat of FIRE II at typical flight condition have been computed and analyzed.

\section{EXPERIMENTAL AND COMPUTATIONAL METHODS}

\section{Experiment Setup and Test Techniques}

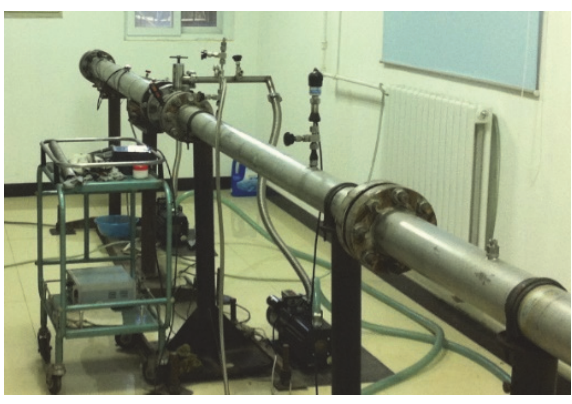

(a)

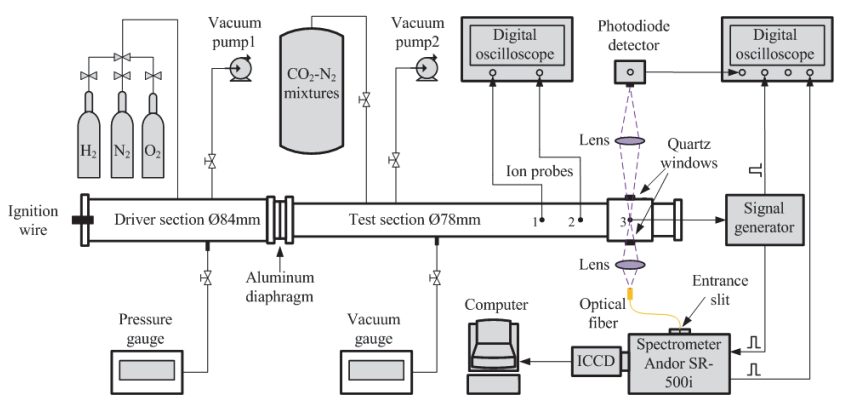

(b)

FIGURE 1. Demonstration of the shock tube and experiment setup

The experiments of high temperature air spectral radiation intensity measurement have been conducted in a combustion driven shock tube of Institute of Mechanics, Chinese Academy of Science. A photograph of the facility is showed in FIGURE 1 (a). The shock tube mainly consists of two parts, the driver section and the test section, which are all circular cross-section stainless iron tube. The diameter of the test section is $78 \mathrm{~mm}$.

FIGURE 1 (b) denotes the experiment setup and diagnostic system. The quartz window is located about $1.5 \mathrm{~m}$ downstream from the aluminum diaphragm that separates two sections. The driver section is filled with $\mathrm{O}_{2}, \mathrm{~N}_{2}$ and $\mathrm{H}_{2}$ to generate the combustion wave to drive the gas flow. $\mathrm{O}_{2}, \mathrm{~N}_{2}$ and $\mathrm{CO}_{2}$ can be filled in the test section to simulate the Earth or Mars atmosphere. By adjusting the mole fraction of $\mathrm{H}_{2}$ and $\mathrm{O}_{2}$ in the driver section and the pressure in the test section, the shock tube can produce shocks with velocity spanning from 2 to $8 \mathrm{~km} / \mathrm{s}$. In the tests, the shock velocity is measured by ion transducers.

The diagnostic system includes lens, optical filters, ICCD and spectrometers. In the test of air emission radiation measurement, $\mathrm{CaF}_{2}$ windows were used. The calibration was accomplished before every 3 or 4 test shots, by setting a tungsten halogen lamp at exactly the same position, to obtain the relation function of the measured counts and absolute radiation.

\section{Equations and Computational Methods}

Gas radiation computation contains 4 main parts ${ }^{[11]}$. The first is flowfield solving to obtain the temperature and species number densities. The second is electronic state population calculation. The third is spectrum calculation to get emission and absorption coefficients in specified frequency range. The last is radiation transfer computation to attain the radiative intensity distribution in space and solid angles. In the computation of spectral emission radiation in shock tube, radiation transfer was neglected.

Navier-Stokes equations with thermo-chemical non-equilibrium models have been solved to simulate the high enthalpy flow in front of re-entry vehicles. Based on finite volume method on multi-block structured grids, van Leer 
scheme was used on convection term, where the interface value was obtained by MUSCL interpolation with van Albada limiter; second-order central scheme was employed on viscous term; third order Runge-Kutta method was adopted in time marching for unsteady computation. 11 species and 20 reactions model of air from Gupta ${ }^{[12]}$ was taken to compute chemical reaction sources. Thermal equilibrium was assumed here, so only 1 temperature was considered. The transportation and thermodynamic parameters were calculated through temperature polynomials.

$$
\begin{aligned}
& \frac{\partial \boldsymbol{U}}{\partial t}+\frac{\partial \boldsymbol{F}}{\partial x}+\frac{\partial \boldsymbol{G}}{\partial y}+\frac{\partial \boldsymbol{H}}{\partial z}=\frac{\partial \boldsymbol{F}_{\boldsymbol{v}}}{\partial x}+\frac{\partial \boldsymbol{G}_{\boldsymbol{v}}}{\partial y}+\frac{\partial \boldsymbol{H}_{\boldsymbol{v}}}{\partial z}+\boldsymbol{S} \\
& U=\left\{\begin{array}{c}
\rho_{i} \\
\rho u \\
\rho v \\
\rho w \\
\rho E
\end{array}\right\} F=\left\{\begin{array}{c}
\rho_{i} u \\
\rho u^{2}+p \\
\rho u v \\
\rho u w \\
u(\rho E+p)
\end{array}\right\} \boldsymbol{G}=\left\{\begin{array}{c}
\rho_{i} v \\
\rho u v \\
\rho v^{2}+p \\
\rho v w \\
v(\rho E+p)
\end{array}\right\} \boldsymbol{H}=\left\{\begin{array}{c}
\rho_{i} w \\
\rho u w \\
\rho v w \\
\rho w^{2}+p \\
w(\rho E+p)
\end{array}\right\} \\
& \boldsymbol{F}_{\boldsymbol{v}}=\left\{\begin{array}{c}
D_{i} \frac{\partial \rho_{i}}{\partial x} \\
\tau_{x x} \\
\tau_{x y} \\
\tau_{x z} \\
\tau_{x x} u+\tau_{x y} v+\tau_{x z} w-q_{x}
\end{array}\right\} \boldsymbol{G}_{\boldsymbol{v}}=\left\{\begin{array}{c}
D_{i} \frac{\partial \rho_{i}}{\partial y} \\
\tau_{y x} \\
\tau_{y y} \\
\tau_{y z} \\
\tau_{y x} u+\tau_{y y} v+\tau_{y z} w-q_{y}
\end{array}\right\} \boldsymbol{H}_{\boldsymbol{v}}=\left\{\begin{array}{c}
D_{i} \frac{\partial \rho_{i}}{\partial z} \\
\tau_{z x} \\
\tau_{z y} \\
\tau_{z z} \\
\tau_{z x} u+\tau_{z y} v+\tau_{z z} w-q_{z}
\end{array}\right\} \boldsymbol{S}=\left\{\begin{array}{c}
\dot{w}_{i} \\
0 \\
0 \\
0 \\
0
\end{array}\right\}
\end{aligned}
$$

A narrowband model was employed to calculate gas radiative characteristics. In these calculations, $\mathrm{N}_{2}$ first positive band, second positive band, $\mathrm{N}_{2}{ }^{+}$first negative band, $\mathrm{NO} \gamma, \beta, \sigma$ and $1100 \mathrm{~nm}$ bands, $\mathrm{O}_{2}$ Shuman-Runge band, $\mathrm{N}$ and $\mathrm{O}$ atomic lines and continuum, and $\mathrm{N}^{+}$and $\mathrm{O}^{+}$continuum radiation were considered based on electronic transition.

The gas absorption coefficients can be calculated by:

$$
k_{\eta}=\frac{8 \pi^{3} \eta_{0}}{3 h c} \frac{N_{e, v, J}^{\prime} R_{e}^{2}\left(\bar{r}_{v^{\prime}, v^{\prime \prime}}\right) q\left(v^{\prime}, v^{\prime \prime}\right) S_{J^{\prime \prime} \Lambda^{\prime \prime}}^{J^{\prime} \Lambda^{\prime}}}{2 J^{\prime \prime}+1}
$$

Where $\eta_{0}$ is central wave number; $h$ denotes Planck constant; $c$ represents optical velocity; $N_{e, v, J}^{\prime}$ is number density of low energy level particles; $R_{e}\left(\bar{r}_{v^{\prime}, v^{\prime \prime}}\right)$ is electronic transition matrix of vibrational energy form $v^{\prime}$ to $v^{\prime \prime} ; q\left(v^{\prime}, v^{\prime \prime}\right)$ is Franck-Condon factor; $v^{\prime}$ and $v^{\prime \prime}$ denote low and high vibrational energy level; $S_{J^{\prime \prime} A^{\prime \prime}}^{I^{\prime}}$ is intensity factor of rotational spectrum; $J$ and $\Lambda$ represent quantum number and degeneracy of rotational energy level; and $F(\eta)$ is spectrum line type function.

Radiation transfer equation is about energy conservation along the optic path. Radiative intensity is function of wavelength, time, space position and solid angle, so it is complicated to solve radiation transfer equation. The spectrum band radiation transfer equation can be written as:

$$
\frac{d I_{k}(l, \omega)}{d l}=\alpha_{k} I_{b k}(l)-\kappa_{k} I_{k}(l, \omega)+\frac{\sigma_{\mathrm{s}, k}}{4 \pi} \int_{4 \pi} I_{k}\left(l, \omega^{m^{\prime}}\right) \Phi_{k}\left(\omega^{m}, \omega^{m^{\prime}}\right) d \omega^{m^{\prime}}
$$

Where $I$ is radiative intensity; $l$ is space position; $\omega$ is solid angle; $\alpha_{k}, \kappa_{k}$ and $\sigma_{s, k}$ are respectively emission, decay and scattering coefficients, $\kappa_{k}=\alpha_{k}+\sigma_{s, k} ; \Phi_{k}$ represents scattering phase function, $\omega_{m}$ and $\omega_{m}$ ' are incidence and scattering angle; subscript $k$ means the $k$-th zone in band model. Scattering can be neglected for gas radiation, so it reduces the equation a lot.

In this paper, radiation transfer equation is discrete and computed using finite volume method as NS equations on the same grid of flow solving, which is convenient for flow radiation coupling. The discrete equation in a control volume can be written as:

$$
\sum_{j=1}^{M_{c}} Q_{k, j}^{m}=\sum_{j=1}^{M_{c}} A_{c, j} I_{k, c, j}^{m} D_{j}^{m} \approx\left[\kappa_{k, P} I_{b k, P}-\kappa_{k, P} I_{k, P}^{m}\right] V_{P} \Omega^{m}
$$

Subscript $j$ denotes the center of the interface surface; $D_{j m}$ is the projection of solid angle on the surface.

Radiative heat is obtained by integrating intensity in directions towards the wall over solid angles.

$$
q_{w}^{r}=\sum_{k=1}^{M_{b}} \varepsilon_{k, w}\left(E_{b k, w}-\sum_{D_{w}^{m}<0} I_{k, w}^{m} \mid D_{w}^{m}\right)
$$




\section{RESULTS AND DISCUSSIONS}

\section{Experiment Results and Validation}

The absolute radiative intensity obtained from the test of air with shock velocity of $8.1 \mathrm{~km} / \mathrm{s}$ is shown in FIGURE 2. The pressure in test section for this shot is $20 \mathrm{~Pa}$. The spectral radiative intensity from $330 \mathrm{~nm}$ to $370 \mathrm{~nm}$ was measured.

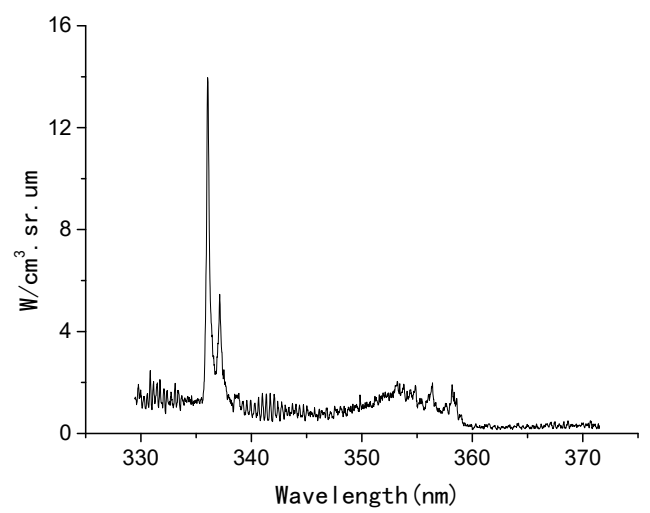

FIGURE 2. Measured volumetric radiative intensity of air at shock velocity $8.1 \mathrm{~km} / \mathrm{s}$.

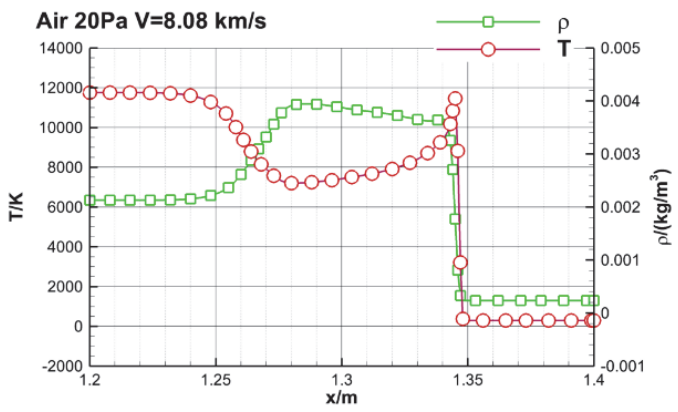

(a) Temperature and density

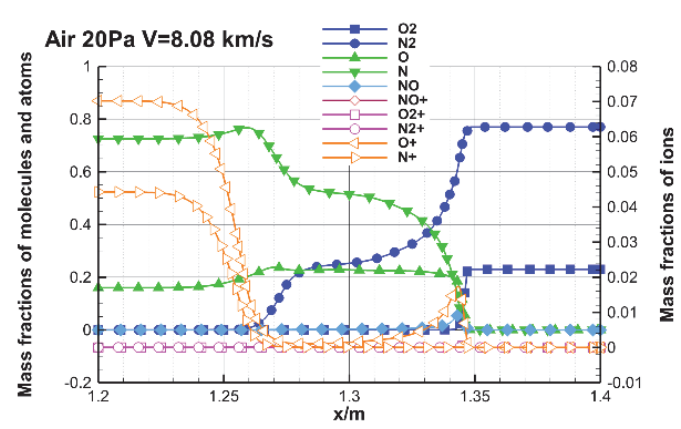

(b) Species mass concentrations.

FIGURE 3. Flow parameters of shock tube simulation.

A corresponding computational simulation has been carried out to generate the shock and the flow which match the test case. In the simulation, the shock velocity was $8.08 \mathrm{~km} / \mathrm{s}$ which was close to the experiment. The distribution of density, temperature, and species mass concentration are shown in FIGURE 3 (a) and (b). The temperature right behind the shock get close to $12000 \mathrm{~K}$ and the temperature in equilibrium region of shock layer is about $8000 \mathrm{~K}$. In this high temperature shock, $\mathrm{O}_{2}$ is completely dissociated and $\mathrm{N}_{2}$ is partly dissociated. $\mathrm{O}^{+}$can be seen clearly in the shock layer comparing to other ion species.

FIGURE 4 is the comparison of radiative intensity between experiment measurement and computational results in the spectral range of 330 to $370 \mathrm{~nm}$. It is noticed that a peak intensity appears at the position of $335 \mathrm{~nm}$, which is considered to be the contamination from $\mathrm{CN}$. Except that, the computed radiative intensity agrees well with experiment data, which validates the flow solver and radiative characteristics calculation of air in high temperature conditions. 


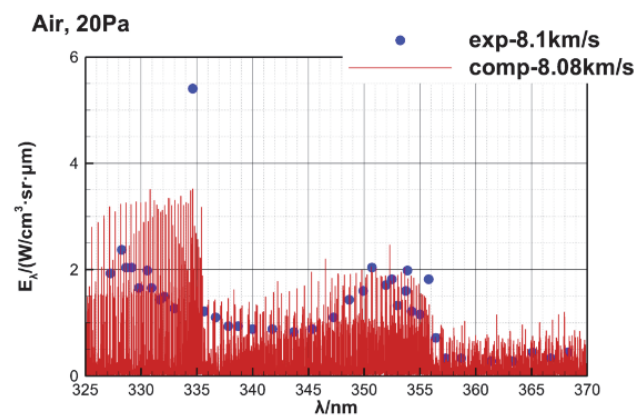

FIGURE 4. Comparison of radiative intensity for the shock velocity $8.1 \mathrm{~km} / \mathrm{s}$ case.

\section{Application on FIRE II}

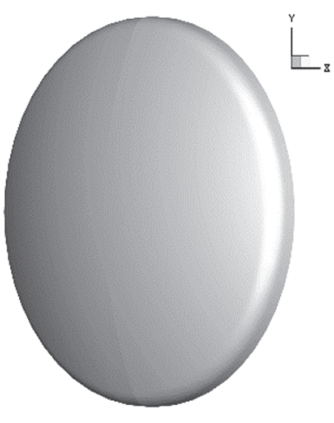

(a) Aeroshell shape

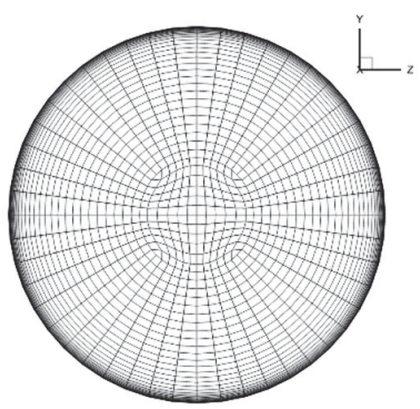

(b) Surface mesh

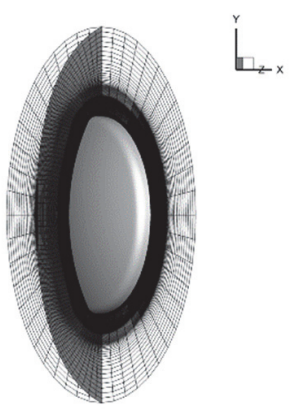

(c) Grids

FIGURE 5. Geometry model and meshes on surface and symmetric plane

FIRE II is an Apollo like re-entry probe. C-H type mesh refined in shock layer has been used in computation as shown in FIGURE 5, in which the minimum grid length normal to the wall was set to $1 \times 10^{-7} \mathrm{~m}$ to guarantee the convergence and accuracy of convective heat prediction.

The peak heating trajectory point that is $1643 \mathrm{~s}$ has been chosen to simulate. The effective radius of the probe at this point equals to $0.747 \mathrm{~m}$. The altitude, velocity, inflow temperature and pressure are $53.04 \mathrm{~km}, 10480 \mathrm{~m} / \mathrm{s}, 276 \mathrm{~K}$ and $7.8 \times 10^{-4} \mathrm{~kg} / \mathrm{m}^{3}$ respectively.

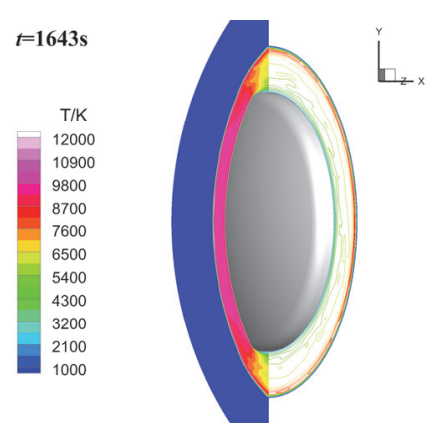

(a) Temperature

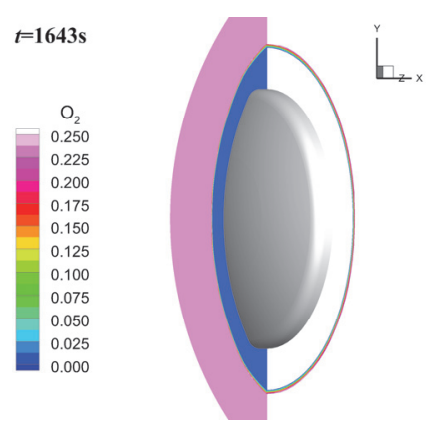

(b) $\mathrm{O}_{2}$ mass fraction

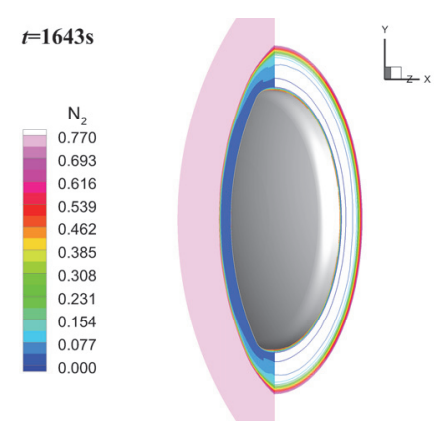

(c) $\mathrm{N}_{2}$ mass fraction

FIGURE 6. Temperature and reactive molecules mass concentrations at $t=1643 \mathrm{~s}$ 
The temperature distribution is shown in FIGURE 6 (a). It can be seen that the temperature exceeds $10000 \mathrm{~K}$ in the whole shock layer because of the extreme high entry velocity. The distribution of reactive molecules, which are $\mathrm{O}_{2}$ and $\mathrm{N}_{2}$, are shown in FIGURE 6 (b) and (c). Unlike that $\mathrm{N}_{2}$ was partly dissociated at $8 \mathrm{~km} / \mathrm{s}$ shock velocity described in former section, $\mathrm{O}_{2}$ and $\mathrm{N}_{2}$ were completely dissociated in the shock layer at $t=1643 \mathrm{~s}$ where the velocity exceeds $10 \mathrm{~km} / \mathrm{s}$.

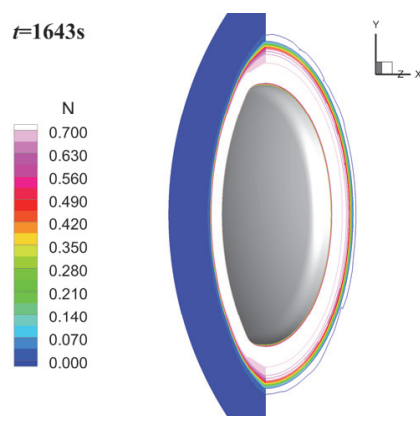

(a) $\mathrm{N}$ mass fraction

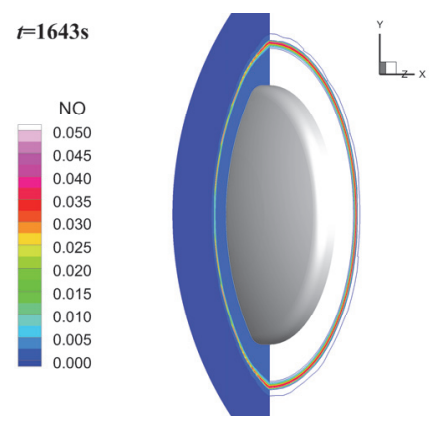

(b) NO mass fraction

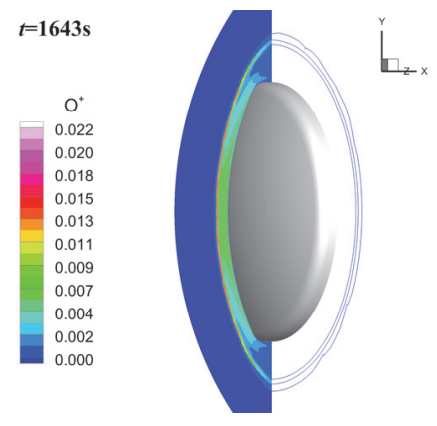

(c) $\mathrm{O}^{+}$mass fraction

FIGURE 7. Productive molecule, atom and ion mass concentrations at $t=1643 \mathrm{~s}$

Some of the productive species distributions are shown in FIGURE 7, including $\mathrm{N}$ in (a), $\mathrm{NO}$ in (b) and $\mathrm{O}^{+}$in (c). In the shock layer, most of $\mathrm{N}_{2}$ were dissociated to atom $\mathrm{N}$. NO were produced near the shock. Not only the neutral species, ions like $\mathrm{O}^{+}$were also produced in shock layer.

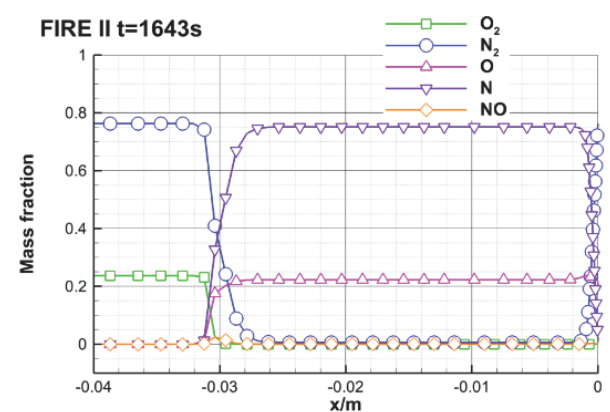

(a) Neutral species mass concentrations

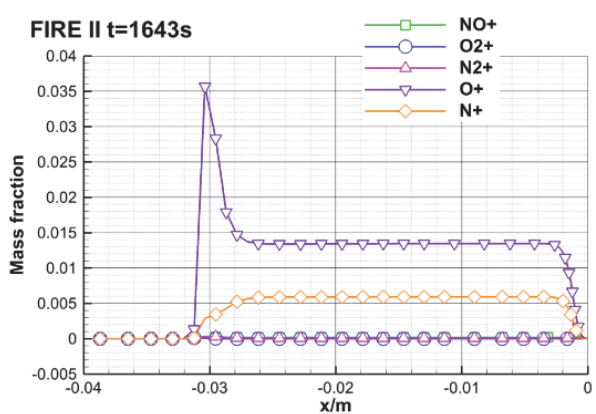

(b) Ions mass concentrations

FIGURE 8. Mass concentrations along the stagnation line at $t=1643 \mathrm{~s}$

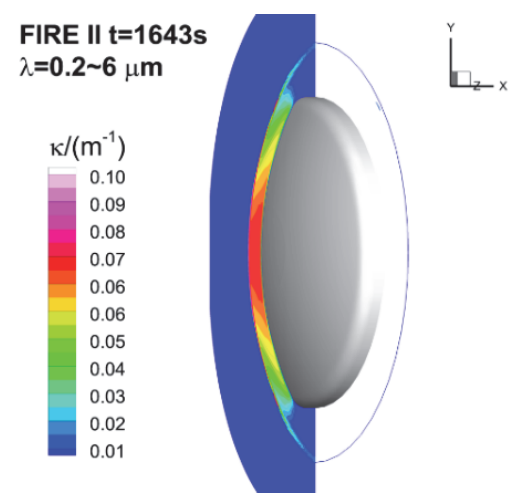

FIGURE 9. Average absorption coefficients at $t=1643 \mathrm{~s}$

The quantitative curve of species mass fraction along the stagnation line are shown in FIGURE 8 . The curves of neutral species in FIGURE 8 (a) denote that there are completely $\mathrm{O}$ and $\mathrm{N}$ in the shock layer, and only a little amount 
of NO near the shock, which is consistent with FIGURE 6 and FIGURE 7. FIGURE 8 (b) shows the curves of ions $\mathrm{O}^{+}$and $\mathrm{N}^{+}$. The distributions of $\mathrm{O}^{+}$and $\mathrm{N}^{+}$are different near the shock, where $\mathrm{N}^{+}$just begin to be produced, but $\mathrm{O}^{+}$ has already reach the peak value which is twice than that in the shock layer. In equilibrium region, mass fraction of $\mathrm{O}^{+}$is about twice than that of $\mathrm{N}^{+}$.

Based on the temperature, pressure and species number density obtained in flowfield solving, absorption coefficients of one specie at the wavelength spanning from $0.2 \mu \mathrm{m}$ to $6 \mu \mathrm{m}$ in one cell unit were obtained. The average absorption coefficients were then calculated by integrating over the whole wave numbers, summing the coefficients of all species, and using Planck average formula. FIGURE 9 shows the distribution of the average absorption coefficients of all species from $0.2 \mu \mathrm{m}$ to $6 \mu \mathrm{m}$, which is similar to the shock structure.

By solving radiation transfer equation, the radiative intensities were obtained based on the average absorption coefficients. Radiative heat was acquired by integrating radiative intensities over all solid angles that were towards the wall. Convective and radiative heat flux on surface are shown in FIGURE 10, where (a) is the results with noncatalytic wall, (b) is the results with full catalytic wall, and (c) is the heating rate at symmetric plane along the radial direction.

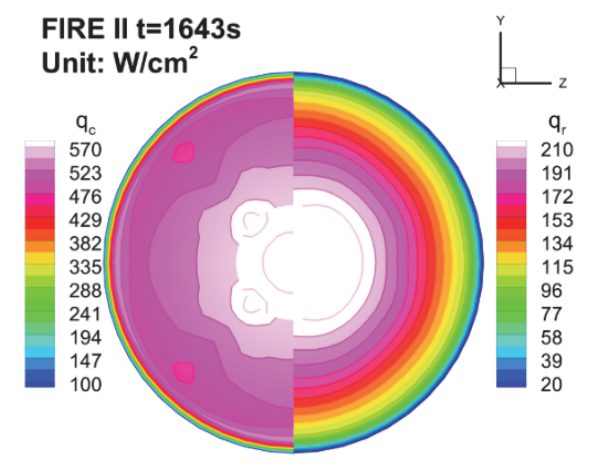

(a) non-catalytic wall

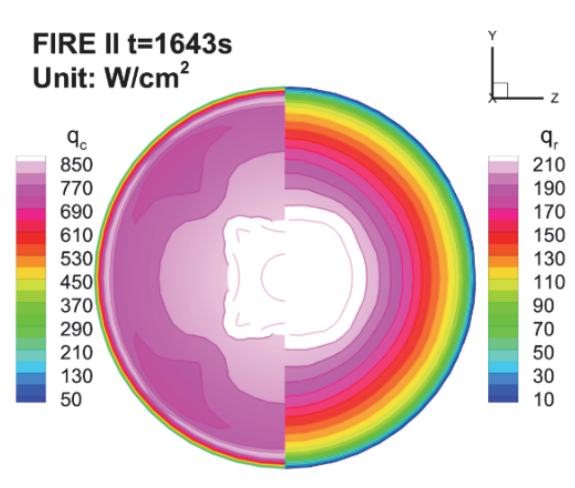

(b) super catalytic wall

FIGURE 10. Convective and radiative heat on surface for both non-catalytic and super catalytic wall

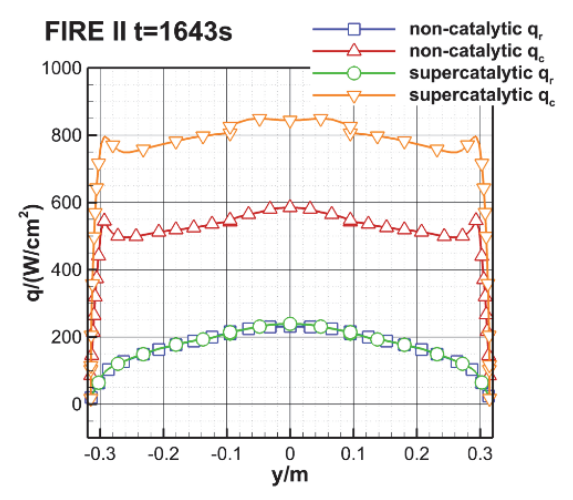

FIGURE 11. Convective and radiative heating rate for both non-catalytic and super catalytic wall

TABLE 1. Comparison of stagnation heating rate between computation and flight data

\begin{tabular}{cccc}
\hline Heating rate $\left(\mathbf{W} / \mathbf{c m}^{2}\right)$ & Super catalytic & Non-catalytic & Flight \\
\hline Radiative heat & 236.2 & 230.0 & - \\
Convective heat & 838.0 & 580.1 & - \\
Total heat & 1074.2 & 810.1 & 1025 \\
\hline
\end{tabular}

The distribution and the value of radiative heating rate with different catalytic wall are almost the same, while convective heating rate with full catalytic wall is $30 \%$ larger than that with non-catalytic wall, as shown in FIGURE 
11. It is noticed that both peak heating from convection and radiation are at stagnation point, but convective heat rises up again on the shoulder. It is believed that the expansion of gas flow after the shoulder leads to the re-compression right before the shoulder, which causes the heating rate grows larger. However, radiative heating rate decreases slowly and monotonously along the radial direction, which is related to the shock thickness and the temperature, not the flow pattern. The first calorimeter for total heating rate measurement of FIRE II was located at $s / R=0.1$, where $s$ denotes the radial distance from heat shield centerline. The convective and radiative heating rate of computation and flight measurement are listed in TABLE 1 . The total heating rate of computation at the point of $s / R=0.1$ is about $816 \mathrm{~W} / \mathrm{cm}^{2}$ and $1083 \mathrm{~W} / \mathrm{cm}^{2}$ for non-catalytic and full catalytic wall respectively. The measured heating rate in flight experiment is $1025 \mathrm{~W} / \mathrm{cm}^{2}{ }^{[13]}$, which is between the value of two simulations.

\section{CONCLUSIONS}

Experiment and computation of flow field and gas radiation of high temperature gas at hyper-velocity conditions have been implemented. Radiation diagnostic system has been built for a combustion driven shock tube. In this facility, absolute gas radiative intensities of air behind $8.1 \mathrm{~km} / \mathrm{s}$ shock have been measured. Simulations under the same conditions have been implemented using an in-house flowfield and radiation solver based on finite volume method. Comparisons show good agreement between the measurement and computation, which validate the numerical models and methods. Then simulations on FIRE II at peak heating trajectory point have been carried out. Convective and radiative heat have been obtained by solving the flow and radiation. At hyper-velocity conditions, reactive molecules as $\mathrm{O}_{2}$ and $\mathrm{N}_{2}$ were completely dissociated, ions as $\mathrm{O}^{+}$and $\mathrm{N}^{+}$were produced in the shock layer. By solving radiation transfer equation, both convective and radiative heat were acquired. It was found that convective heat with noncatalytic and full catalytic wall had same distributions, but different values. However, radiative heating rate were approximately the same with both catalytic wall conditions. It was also noticed that radiative heating rate decreased slowly and monotonously along the radial direction, while convective heat rose up again on the shoulder. Total heating rate of two wall models at peaking trajectory point covered the flight data.

\section{ACKNOWLEDGMENTS}

The authors gratefully acknowledge the support by the National Natural Science Foundation of China (Grant No.: $11772315,11402251)$.

\section{REFERENCES}

1. J. D. Anderson, AIAA Journal 7(9), 1665-1675 (1969).

2. D. L. Cauchon, "Radiative Heating Results from the Fire II Flight Experiment at a Reentry Velocity of 11:4 km/s," NASA TM X-1402 (1967).

3. E. S. Cornette, "Forebody temperature and calorimeter heating rates measured during project FIRE II reentry at 11.35 km/s," NASA TM X-1305 (1966).

4. I.D. Boyd and P.M. Jenniskens, Journal of Spacecraft and Rockets 47(6), 901-909 (2010).

5. S. Abe et al, Publications of the Astronomical Society of Japan 63(5), 1011-1021 (2011).

6. P. Reynier, Progress in Aerospace Sciences 85, 1-32 (2016).

7. A.M. Brandis and B.A. Cruden, "Benchmark EAST experiments for earth re-entry," in Proc. of the 55th AIAA Aerospace Sciences Meeting, (AIAA, Grapevine, Texas, 2017), 1145.

8. A.M. Brandis et al, "Non-equilibrium Radiation for Earth Entry," in Proc. of the 46th AIAA Thermophysics Conference, (AIAA, 2016), 3690.

9. S.W. Lewis et al, Journal of Spacecraft and Rockets. 53(5), 887-899 (2016).

10. H. Takayanagi, K. Fujita, "Absolute radiation measurements behind strong shock wave in carbon dioxide flow for mars aerocapture missions," in Proc. of the 43rd AIAA Thermophysics Conference, (AIAA, 2012 ), 2744.

11. C.O. Johnston, "Nonequilibrium shock-layer radiative heating for earth and titan entry," Ph.D. thesis, Virginia Polytechnic Institute and State University, Blacksburg, VA ,2006.

12. R.N. Gupta et al, "A review of reaction rates and thermodynamic and transport properties for an 11-species air model for chemical and thermal nonequilibrium calculations to $30000 \mathrm{~K}$," NASA STI/Recon Technical Report N, 90, 27064 (1990).

13. G. E. Palmer, T. White and A. Pace, Journal of Spacecraft and Rockets 48(5), 836-845 (2011). 